\title{
On-Demand Deposition of Functional Oxide Microdots by Double-Pulse Laser-Induced Dot Transfer
}

\author{
Aiko NARAZAKI ${ }^{* 1}$, Ryozo KUROSAKI ${ }^{* 1}$, Tadatake SATO*1, Yoshizo KAWAGUCHI ${ }^{* 1}$ and Hiroyuki NIINO*1 \\ ${ }^{* 1}$ Research Institute for Innovation in Sustainable Chemistry, National Institute of Advanced Indus- \\ trial Science and Technology (AIST), Central 5, 1-1-1 Higashi, Tsukuba, Ibaraki 305-8565, Japan \\ E-mail:narazaki-aiko@aist.go.jp
}

\begin{abstract}
We have newly developed a double-pulse laser-induced dot transfer technique to realize ondemand deposition of functional oxide microdots under room-temperature atmospheric conditions. In our double-pulse system, the first pulse was irradiated to preheat an oxide source film, and then the second pulse was more tightly focused on the same position to deposit an oxide microdot onto a receiver substrate. As a model case, indium tin oxide microdots with much smaller lateral dimensions than the laser focal area were reproducibly arrayed on a silica glass substrate by the doublepulse process, while there were microdot vacancies at a rate of approximately $30 \%$ in the case of a single-pulse process without preheating. In order to explore the effect of double-pulse, laser-induced temperature distribution was also investigated from a finite elemental approach. DOI: $10.2961 / \mathrm{j} l \mathrm{mn} .2014 .01 .0003$
\end{abstract}

Keywords: double-pulse laser-induced dot transfer, oxide microdot, micro-patterning, microdot vacancy, finite element method

\section{Introduction}

Laser-induced transfer is an attractive additive micropatterning method because it has the advantage of being a simple atmospheric room-temperature process [1-3]. Recently, micro-patterning of metal [4-7] and semiconducting [8-10] microdots has been reported using laser-induced transfer with one-to-one dot deposition and sub-spot resolution, which is referred to as laser-induced dot transfer (LIDT) [8-10] to distinguish it from conventional laser transfer techniques. In LIDT, a single laser pulse is tightly focused onto a source film, which is on a supporting plate, and site-controlled deposition of a single melt droplet onto a receiver substrate placed behind the film occurs. The droplets have much smaller lateral dimensions than the laser focal area. The LIDT process is advantageous in that it has higher resolutions than commercially available inkjet techniques and there is an availability of solvent-free source materials.

There is an additional attractive point of LIDT; the transferred materials are able to have a pseudo-sphere due to the surface tension of the micrometer-sized melt droplets [4-10]. For example, transparent microspheres are promising components for compact optical circuits such as $90^{\circ}$ bend optical waveguides and photonic routers because these microspheres can confine light in a small volume due to the optical whispering gallery mode $[11,12]$. In the whispering gallery mode, the light waves are almost perfectly guided round by optical total internal reflection, leading to high $\mathrm{Q}$ factors. Templates for nanohole fabrication can be created using the near-electromagnetic field around such microspheres [13]. Thus, a simple on-demand method of aligning transparent microspheres, such as
LIDT, will be a key technology for compact optical and optoelectronic integrations.

However, in the case of LIDT of oxide materials, it is somewhat difficult to deposit oxide microdots with a diameter smaller than the laser focal spot size [9, 10]. Furthermore, oxide films often fracture after laser irradiation, emitting film flakes onto the receiver substrate and degrading the micro-patterns. Recently, we made detailed examination of single-pulse LIDT of oxide materials with varying film thickness, leading to transfer of oxide microdots with a spherical shape [14].

In this paper, we report a novel double-pulse LIDT process in order to improve micro-patterning of oxide microdots without dot vacancies. Different from metal microdots [4-7], single-pulse LIDT of oxides easily tends to make dot vacancies when they are arrayed in micro-patterns. To overcome this problem, we have developed a double-pulse LIDT process that is the combination of the first pulse for preheating and the second pulse for dot transfer. To investigate laser-induced temperature distribution during the double-pulse LIDT, the transient temperature in the ITO source film was also simulated using finite element method (FEM) techniques.

\section{Experimental}

Figure 1 schematically shows our double-pulse LIDT system. Two types of light sources operated at the same laser wavelength of $266 \mathrm{~nm}$ are used. The first laser pulse from a laser source I is irradiated onto a transparent support / oxide source film interface through the support with a larger focal beam size to preheat the film. After adequate time delay $t_{\mathrm{d}}$, the second laser pulse from another laser source II is more tightly focused on the same position for 


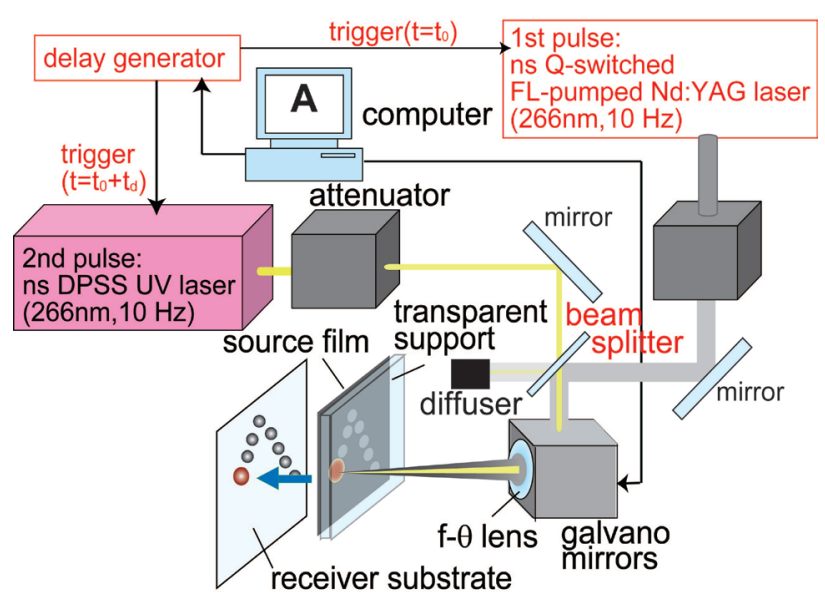

Fig. 1 Setup for the on-demand patterning of functional oxide microdots by double-pulse laser-induced dot transfer. The first laser pulse is irradiated onto a transparent support / oxide source film interface through the support to preheat the film. After adequate time delay $t_{\mathrm{d}}$, the second laser pulse is more tightly focused on the same position for microdot transfer.

microdot transfer. In this work, a flashlamp-pumped $\mathrm{Nd}$ :YAG pulsed laser (Spectra-Physics, INDI; $\lambda=266 \mathrm{~nm}$, full width at half maximum of $7 \mathrm{~ns}$, multimode, maximum frequency of $10 \mathrm{~Hz}$ ) was used as the laser source I. The laser source II was a Nd:YVO ${ }_{4}$ DPSS laser (Coherent, AVIA $266 ; \lambda=266 \mathrm{~nm}$, full width at half maximum of 30 ns, $\left.M^{2}<1.3\right)$. The focal size at the interface between the film and support was $80 \mu \mathrm{m}$ for the first pulse and $20 \mu \mathrm{m}$ for the second pulse, respectively. The time delay, $t_{\mathrm{d}}$ was controlled by trigger signals to both light sources using a delay generator. These lasers were operated at a repetition rate of $10 \mathrm{~Hz}$ due to the limitation of the laser source $\mathrm{I}$ in this work. Consecutive double pulses were scanned with a galvanometer-based point-scanning module (GSI Lumonics, HPM10M2) for on-demand microdot deposition. For comparison, a single-pulse LIDT was also conducted using only a single pulse from the laser source II without preheating.

ITO source films with a thickness of $250 \mathrm{~nm}$ were prepared on the silica glass plates by RF magnetron sputtering. An ozone-cleaned silica glass plate was placed as a receiver substrate behind the ITO film at a distance of about 100 $\mu \mathrm{m}$ using a spacer. After double-pulse irradiation at optimum fluence, a single microdot is deposited onto the receiver substrate. The surface morphologies of the deposited-microdot samples were observed using a scanning electron microscope (SEM; Keyence, VE-7800) and a confocal scanning laser microscope (Keyence, VK-8500). Crosssectional profiles were also obtained by laser microscopy.

Using the nanosecond laser pulse to heat a material, the energy transfer is governed by the heat diffusion equation, which is based on Fourier's law of conduction:

$$
\rho c_{p} \frac{\partial T}{\partial t}=k \nabla^{2} T+\dot{q}
$$

where $\rho, c_{p}, k$ and $\dot{q}$ are density, specific heat, thermal conductivity and a rate of heat generation due to the laser light absorption, respectively.

Since the laser pulse through the silica glass support is absorbed in the ITO film, the heat diffusion equation can be separated into two equations for the absorbing ITO film and the transparent silica glass support:

$$
\begin{aligned}
& \rho_{f} c_{p f} \frac{\partial T_{f}}{\partial t}=k_{f} \nabla^{2} T_{f}+\dot{q} \\
& \dot{q}=-\left(1-R_{1}\right)\left(1-R_{2}\right) \frac{d I(z, t)}{d z} \\
& \quad=\left(1-R_{1}\right)\left(1-R_{2}\right) \alpha I_{0}(t) e^{-\alpha z} \quad 0 \leq t \leq t_{p} \\
& \rho_{s} c_{p s} \frac{\partial T_{s}}{\partial t}=k_{s} \nabla^{2} T_{s}
\end{aligned}
$$

where the subscripts of $f$ and $s$ indicate the regions in the film and support, respectively. $R_{1}$ and $R_{2}$ are the reflectivity at air/glass and glass/film interfaces, respectively. $I_{0}(t)$ is the local laser power density at the glass surface and $t_{p}$ is the irradiation time, namely the length of the laser pulse. $\alpha$ is the absorption coefficient of the ITO film.

FEM simulations of the temporal high-temperature distributions in the ITO source films were performed using Ansys (version 12.0). A three-dimensional finite element model with a diameter of $240 \mu \mathrm{m}$ and a height of $90 \mu \mathrm{m}$ was developed to simulate the silica glass/ITO film interface as shown in Fig. 2. Since the model was symmetric and subjected to a symmetric laser pulse that is both spatially and temporally Gaussian in shape, only one half of the cross-sectional plane along the incident laser direction was considered for computational efficiency. The ITO film has a light absorption coefficient of $7.2 \times 10^{6} \mathrm{~m}^{-1}$ at a wavelength of $266 \mathrm{~nm}$ at room temperature. The temperature dependence of the light absorption coefficient of ITO is unknown to the authors, and is neglected in the calculation in this work. The absorption coefficient of solid ITO is

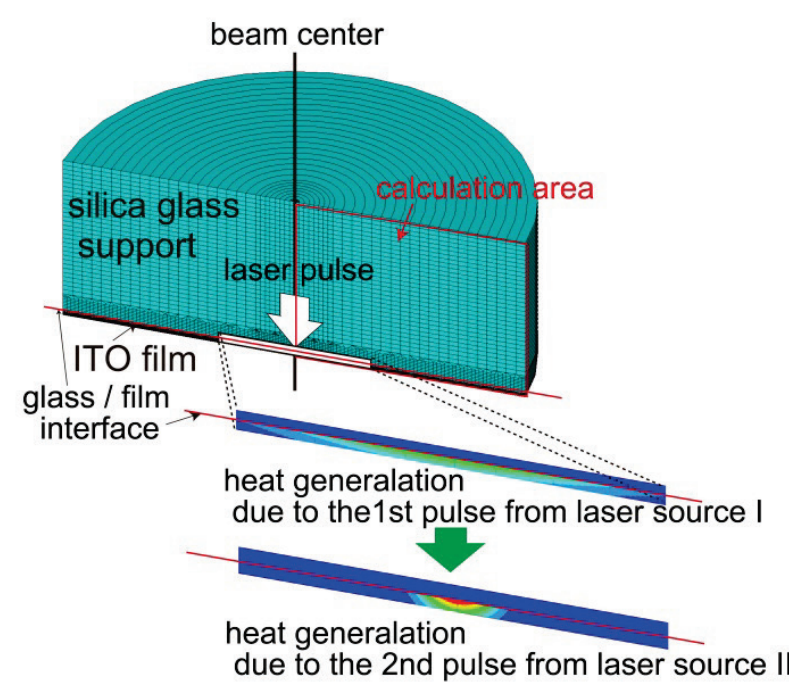

Fig. 2 FEM mesh for the ITO source film on the silica glass transparent support irradiated by double-pulse. The laser pulse is absorbed only in the ITO film and becomes a heating source. 
used for liquid. In addition to Eq. (2), the heat radiation from the surface was taken into account for the ITO film. The emissivity was assumed to be an average value of 0.5 . To simplify the simulation, the silica glass support and ITO source film were assumed to be isotropic, and only the ITO phase change from solid to melt is taken into account as an enthalpy change of $83.74 \mathrm{~kJ} \cdot \mathrm{mol}^{-1}$ around the melting tem-

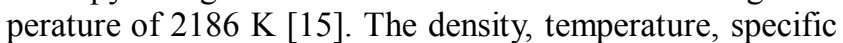
heat, and thermal conductivity values of ITO and silica glass used in our simulation are listed in Table 1 [15-17]. The diameters of first and second laser pulses and the time delay between them used for FEM simulations are set to be same with the experimental conditions in this work.

Table 1 The physical properties of ITO and silica glass.

\begin{tabular}{lcrrr}
\hline & $\begin{array}{c}\rho \\
\left(\mathrm{kg} \cdot \mathrm{m}^{-3}\right)\end{array}$ & \multicolumn{1}{c}{$\begin{array}{c}\mathrm{K}) \\
\end{array}$} & $\begin{array}{c}c_{p} \\
\left(\mathrm{~J} \cdot \mathrm{kg}^{-1} \cdot \mathrm{K}^{-1}\right)\end{array}$ & $\begin{array}{c}k \\
\left(\mathrm{~W} \cdot \mathrm{m}^{-1} \cdot \mathrm{K}^{-1}\right)\end{array}$ \\
\hline ITO $^{\text {a }}$ & \multirow{3}{*}{7180} & 1000 & 362 & \\
& & 2000 & 463 & 5.95 \\
& & 300 & 693 & \\
\hline Silica glass & & 500 & 1049 & 1.38 \\
& 2200 & 1200 & 1130 & 1.62 \\
& & 1600 & 1249 & 4.17 \\
& & &
\end{tabular}

a) The density, $\rho$, and specific heat, $c_{p}$, substitute available values of $\mathrm{In}_{2} \mathrm{O}_{3}$ for those of ITO.

\section{Results and discussion}

\subsection{ITO microdot deposition by double-pulse LIDT}

Figure 3 shows the laser pulse energy dependence of the morphology of the ITO structures transferred by LIDT with double-pulse (red circle) in this work and single-pulse (black circle) in our previous work [14]. In the case of the single-pulse LIDT, there was no transfer below a laser en-

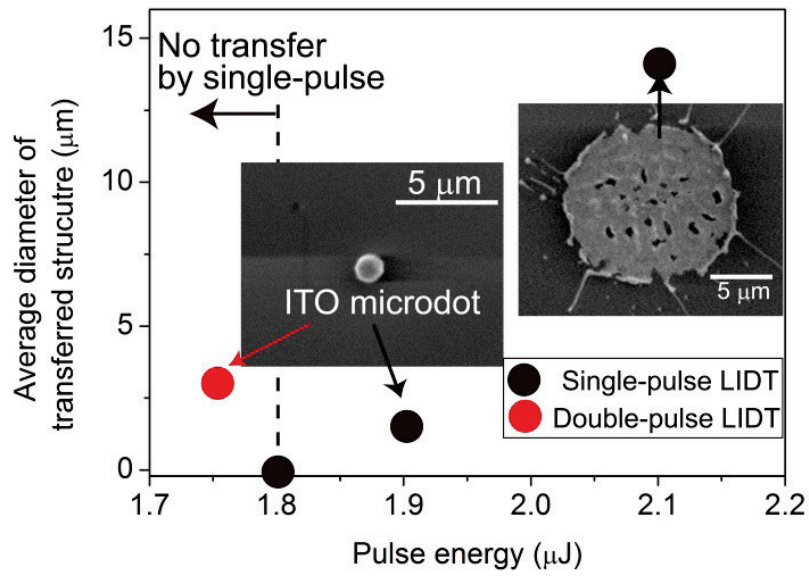

Fig.3 Laser energy (laser source II) dependence of size of ITO microstructures transferred by a single-pulse and double-pulse LIDT. The left and right inset SEM images correspond to an ITO microdot and a disk-like microstructure made by the single-pulse LIDT, respectively. ergy of $1.80 \mu \mathrm{J} \cdot$ pulse $^{-1}$. On the other hand, it was confirmed that the double-pulse LIDT successfully transferred an ITO microdot with a semi-spherical shape at a laser energy of $1.75 \mu \mathrm{J} \cdot$ pulse $^{-1}$. This is because preheating an ITO source film by the first pulse helps to reduce the threshold energy of the second pulse for microdot transfer. It is noteworthy that the morphology of the transferred ITO microstructure changed dramatically from a micrometersized sphere to a disk-like structure with a lateral diameter of $20 \mu \mathrm{m}$, which corresponds to the laser spot size for the single-pulse LIDT. The disk-like structure appears to have solidified after splashing on the receiver substrate, indicating that the higher pulse energy induced significant melting and partial vaporization of the ITO source film that prevented the transferred material from landing softly on the substrate. This kind of laser energy dependence is similar to that seen for commonly used metallic materials and indicates that the laser pulse energy is a vital factor in controlling the LIDT process.

Figures 4 (a) and (b) show confocal scanning laser microscopic images and profile of ITO microdot arrays fabricated by double-pulse and single-pulse LIDT, respectively.
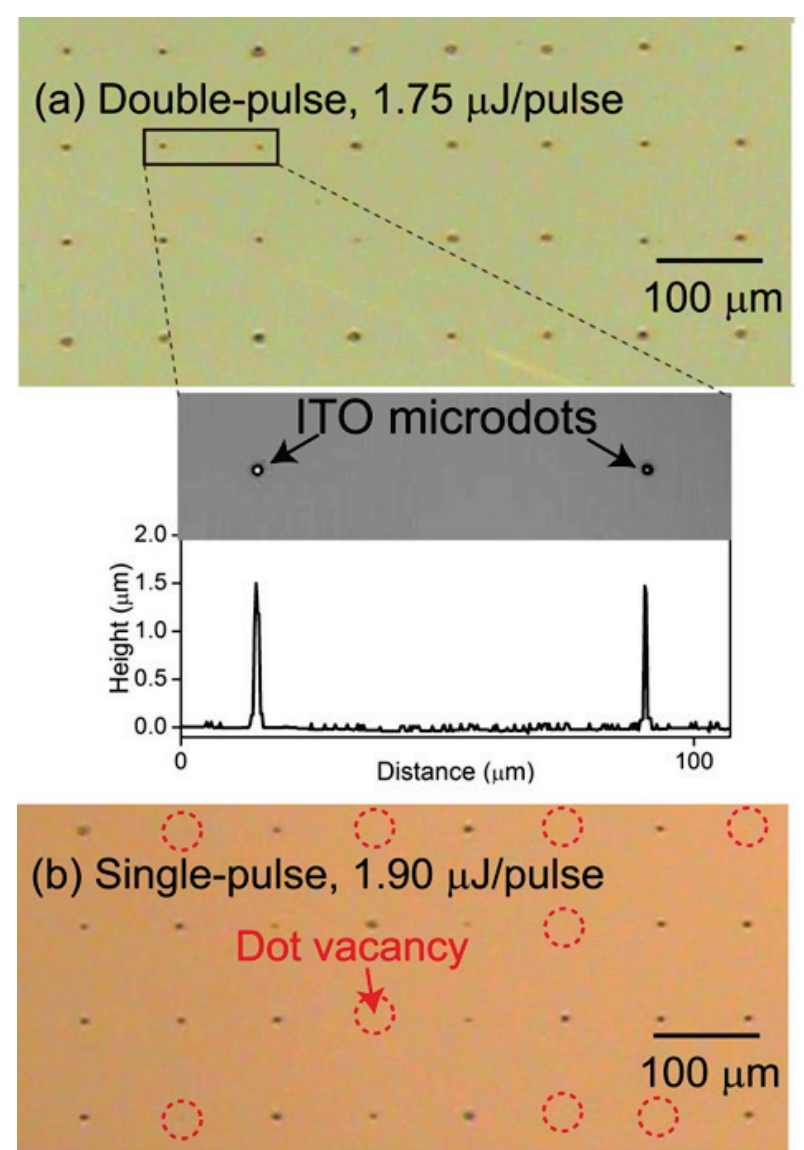

Fig.4 Confocal scanning laser microscopic images and profile of the ITO microdot arrays deposited by (a) double-pulse and (b) single-pulse LIDT, respectively. The time delay, $t_{\mathrm{d}}$ was $500 \mathrm{~ns}$. The ITO microdots with a height of $1.5 \mu \mathrm{m}$ were reproducibly arrayed by the double-pulse LIDT, whereas there were dot vacancies (red dotted circles) at a rate of approximately $30 \%$ for the single-pulse LIDT. 


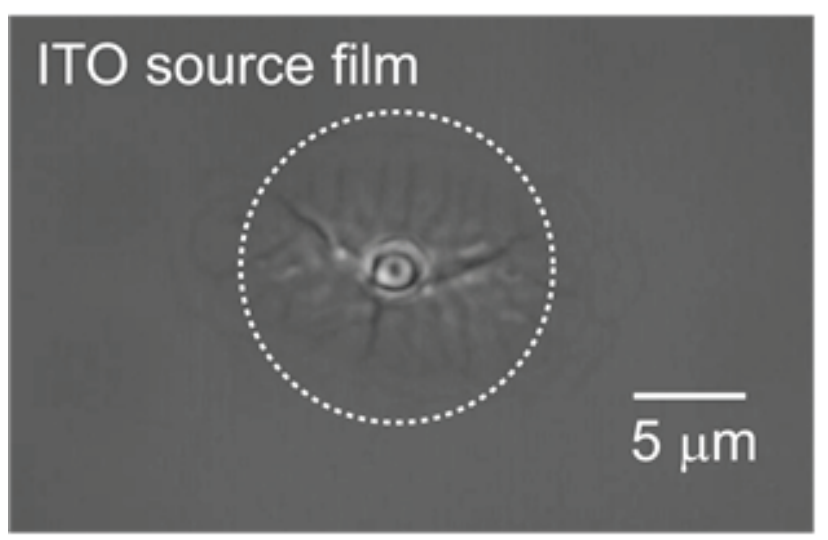

Fig.5 A SEM image of the ITO source film after a single-DPSS laser pulse irradiation when no microdot transfer occurred onto a receiver substrate. The white circle denotes a laser focal point. In the circle, a laser-induced microstructure containing a microdot at the center was observed.

In the case of the double-pulse LIDT corresponding to Fig. 4 (a), the laser energy of the first and second pulse was approximately 5 and $1.75 \mu \mathrm{J} \cdot$ pulse $^{-1}$, respectively. For the single-pulse LIDT, the laser energy was $1.90 \mu \mathrm{J} \cdot$ pulse $^{-1}$. ITO microdots with a height of approximately $1.5 \mu \mathrm{m}$ are reproducibly arrayed in Fig. 4 (a). On the other hand, there were microdot vacancies (red dotted circles) at a rate of approximately $30 \%$ for the single-pulse LIDT.

Figure 5 shows a SEM image of an ITO source film after a single-pulse LIDT process when no microdot transfer, namely a dot vacancy, was observed on a receiver substrate. The white circle denotes a laser focal point. In the circle, the film surface morphology changed to a laserinduced microstructure containing a microdot at the center. This result suggests that the laser energy was not enough for transfer of a microdot onto a receiver substrate. The microdot vacancy is often observable when the laser energy is not enough for a dot transfer. Thus, we also tried singlepulse LIDT at the laser energy, which is higher than that for microdot deposition in Fig. 4 (b) and lower than that for transfer of disk-like structures as shown in Fig. 3. As a result, an ITO microdot array was a mixture of microdots and disk-like structures due to pulse-to-pulse laser energy fluctuation. It seems difficult to make oxide microdot arrays without microdot vacancies in the case of single-pulse LIDT of oxide materials because transfer of oxide microdots is sensitive to the laser pulse energy. Therefore, the double-pulse LIDT is effective for functional oxide microdot patterning.

\subsection{Laser-induced temperature distribution in ITO source film by double-pulse laser irradiation}

Finally, we discuss the laser-induced transient temperature in the ITO source film for a typical case of doublepulse LIDT. Figures 6 (a) and (b) show the FEM simulated temperature distributions in the ITO film at 50 ns and 513 ns after the incident first laser pulse, respectively. Figure 6 (c) shows the temporal change in the ITO film temperature at both the film/glass interface and the film surface. The timing of the first pulse (green dotted curve) and the second pulse (black dotted curve) is also shown in fig. 6 (c). The laser pulse energy of the first pulse and second pulse was set at 5 and $1.75 \mu \mathrm{J} \cdot$ pulse $^{-1}$, respectively. After the first pulse irradiation, the film was rapidly preheated up to about $1700 \mathrm{~K}$ at the beam center which was below the ITO melting temperature of $2186 \mathrm{~K}$. In fact, there was no drastic change in film surface morphology after only the first pulse irradiation in ITO source films employed in this work. Then, the film temperature was slowly decreased with time but kept almost heated until the second pulse incidence. As shown in Fig. 6 (a), thus-preheated area covered a focal size of the first pulse. After the incidence of the second pulse, only a center part of the preheated area, correspond-
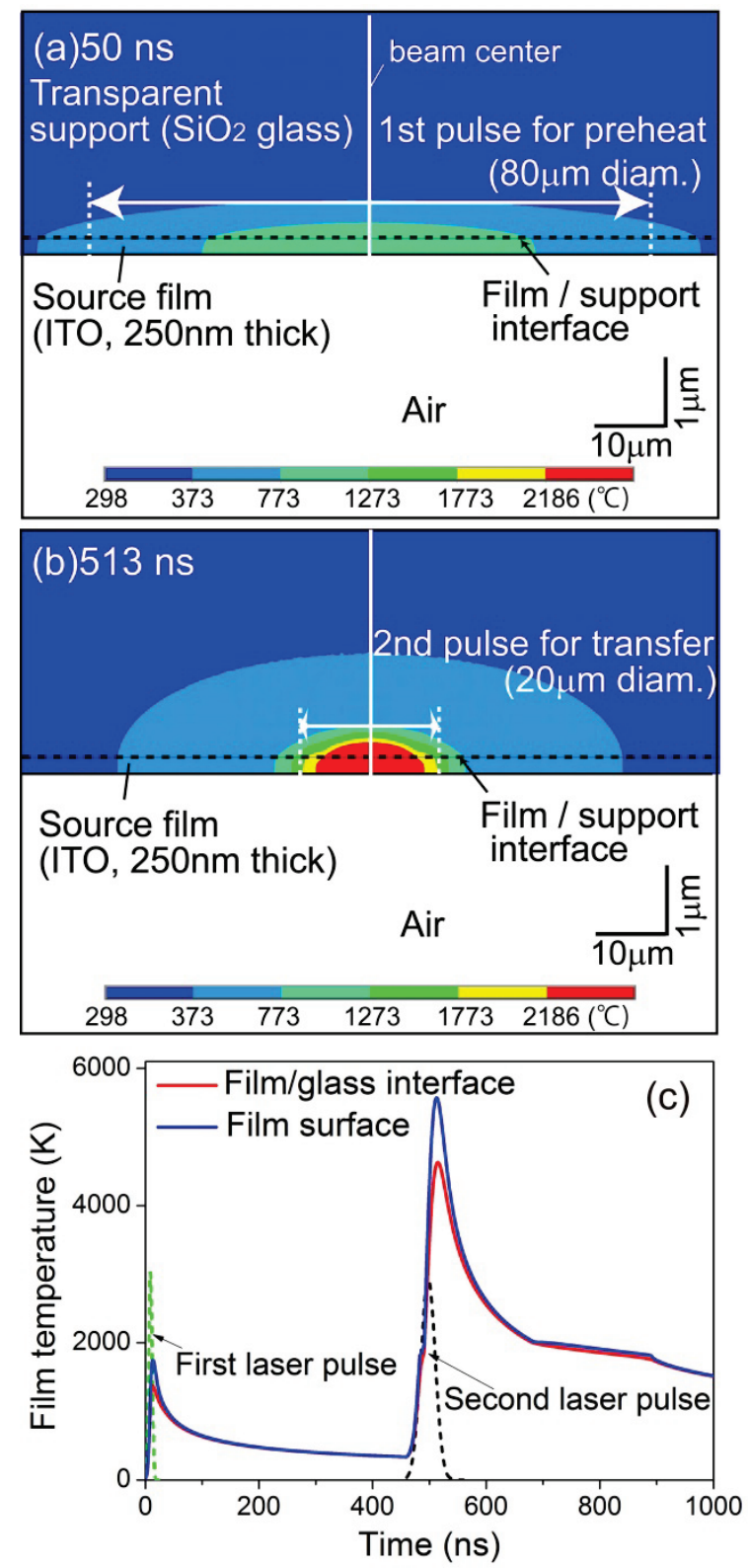

Fig.6 Distribution and temporal change of the ITO film temperature simulated by FEM. (a) The temperature distribution at $50 \mathrm{~ns}$ after the first laser pulse irradiation for pre-heating. (b) The temperature distribution at $513 \mathrm{~ns}$ when the temperature at a film/glass interface reached a maximum. (c) Temporal change of the ITO film temperature at the film/glass interface (red curve) and film surface (blue curve). The timing of the first pulse (green dotted curve) and the second pulse (black dotted curve) is also shown 
ing to the second pulse beam diameter, was reheated and was melted. As a result, the transfer of a single droplet might be achieved like in the LIDT process. Consequently, double-pulse LIDT has a lower threshold of laser pulse energy for transfer compared with that for single-pulse LIDT, as shown in Fig. 3. It is noteworthy that the film temperature at the interface was transiently lower than that at the surface due to heat conduction to the adjacent glass support.

Another effect of double-pulse irradiation might be to be able to reduce thermal stress due to large temperature difference caused by a laser pulse for transfer. In the case of single-pulse LIDT of oxide materials, a fracture of an oxide source film easily occurs and degrades transferred patterns by film fragments, making a narrower range of the optimum laser pulse energy for dot transfer. The behavior of oxides is thus different from most metals, which are ductile and rarely fracture. On the other hand, preheating of large area by the first pulse is effective for reduction of temperature differences in the film. Based on these results, the double-pulse LIDT is vital for the formation of highquality micro-patterns of oxide microdots without dot vacancies.

\section{Conclusion}

We have developed on-demand additive micropatterning of oxide microdots using a novel double-pulse LIDT technique. In our double-pulse LIDT process, the first pulse preheated an oxide source film widely. After an adequate time delay, the second pulse was more tightly focused onto the film through a transparent support, ejecting a single oxide microdot onto the receiver substrate. As a model case, ITO microdots with a height of approximately $1.5 \mu \mathrm{m}$ were successfully arrayed on a silica glass receiver substrate. Different from LIDT with only a single pulse without preheating, a threshold of laser pulse energy for microdot transfer was reduced. Furthermore, the ITO microdots were reproducibly prepared by the double-pulse LIDT, whereas dot vacancies were observed at a rate of approximately $30 \%$ for the single-pulse LIDT. Based on FEM simulations of the laser-induced transient temperature in the ITO source film, the first pulse with a large diameter was effective for the reduction of the threshold energy for microdot transfer as well as to decrease the thermal stress caused by the laser pulse for transfer. Consequently, ondemand deposition of transparent functional micrometersized oxide dots can be realized by double-pulse LIDT with high quality, and it is useful for future compact optical and optoelectronic integrations.

\section{Acknowledgment}

This work was supported by the Fund for Young Researchers, Ministry of Education, Culture, Sports, Science and Technology, Japan.

\section{References}

[1] J. Bohandy, B. F. Kim and F. J. Adrian: J. Appl. Phys., 60, (1986) 1538.

[2] A. Piqué, D. B. Chrisey, R. C. Y. Auyeung, J. FitzGerald, H. D. Wu, R. A. McGill, S. Lakeou, P. K. Wu, V. Nguyen and M. Duignan: Appl. Phys. A, 69, (1999) S279.

[3] Y. Nakata and T. Okada: Appl. Phys. A, 69, (1999) S275.

[4] D. A. Willis and V. Grosu: Appl. Phys. Lett., 86, (2005) 244103.

[5] D. P. Banks, C. Grivas, J. D. Mills, R. W. Eason and I. Zergioti: Appl. Phys. Lett., 89, (2006) 193107.

[6] A. I. Kuznetsov, R. Kiyan and B. N. Chichkov: Opt. Express, 18, (2010) 21198.

[7] A. I. Kuznetsov, C. Unger, J. Koch and B. N. Chichkov: Appl. Phys. A, 106, (2012) 479.

[8] A. Narazaki, T. Sato, R. Kurosaki, Y. Kawaguchi and H. Niino: Appl. Phys. Express, 1, (2008) 57001.

[9] A. Narazaki, T. Sato, R. Kurosaki, Y. Kawaguchi and H. Niino: Appl. Surf. Sci., 255, (2009) 9703.

[10] A. Narazaki, R. Kurosaki, T. Sato, Y. Kawaguchi, W. Watanabe and H. Niino: J. Laser Micro/Nanoengineering. 7, (2012) 77.

[11] Y. Hara, T. Mukaiyama, K. Takeda and M. KuwataGonokami: Phys. Rev. Lett., 94, (2005) 203905.

[12] K. J. Vahala: Nature, 424, (2003) 839.

[13] T. Sakai, N. Nedyalkov, and M. Obara: J. Phys. D, 40, (2007) 2102.

[14] A. Narazaki, R. Kurosaki, T. Sato and H. Niino: Appl. Phys. Express, 6, (2013) 92601.

[15] G. V. Samsonov: “The Oxide Handbook" (Plenum, New York, 1982) pp. 54, 61, 101.

[16] "Thermophysical Properties Handbook" ed. by Japan Society of Thermophysical Properties (Yokendo, Japan, 2008) pp. 272, 300 (in Japanese).

[17] T. Ashida, A. Miyamura, N. Oka, Y. Sato, T. Yagi, N. Taketoshi, T. Baba and Y. Shigesato: J. Appl. Phys., 105, (2009) 073709.

(Received: July 22, 2013, Accepted: December 14, 2013) 